\title{
化学镀镍层对直接生长碳纳米管薄膜强流脉冲发射 稳定性的影响
}

曾凡光 ${ }^{(1)}$, 李昕 ${ }^{(2)}$, 刘卫华 ${ }^{(2)}$, 乔淑珍 ${ }^{(1)}$, 麻华丽 ${ }^{(1)}$, 张锐 ${ }^{(1)}$, 夏连胜 ${ }^{3}$, 谌怡 ${ }^{(3)}$, 刘星光 ${ }^{3}$, 张筀 ${ }^{(3)}$

(1) 郑州航空工业管理学院数理系, 郑州 450015 ;

(2) 西安交通大学电子与信息工程学院, 西安 710049;

(3) 中国工程物理研究院流体物理研究所, 绵阳 621900

E-mail: fgzeng@sina.com

2011-02-18 收稿, 2011-04-15 接受

国家自然科学基金(51072184, 50972132, 51002143, 60801022)、航空科学基金(2009ZE55003, 2010ZF55013)和河南省基础与前沿技术计划 (092300410139)资助

摘要 采用酞菁铁高温热解方法分别在镀镍和不镀镍硅基底上生长了碳纳米管(carbon nanotube, CNT) 薄膜. 镍镀层采用化学镀方法制备. 为了研究有镍层和无镍层 CNT (Si-CNT 和 $\mathrm{Ni}-\mathrm{CNT}$ ) 薄膜阴极的强流脉冲发射稳定性, 在相同的主Marx电压下采用二极结构对 2 种阴极进 行重复发射实验. 当峰值脉冲电压在 1.62 1.66 MV(对应场强为 11.57 11.85 V/ $\mu \mathrm{m}$ ) 时, Si-CNT 和 Ni-CNT 阴极首次发射的电流峰值分别为 109.4 和 180.5 A. 通过比较 2 种阴极的归一化电流, 可明显发现 2 种阴极发射稳定性的差异. Si-CNT 和 Ni-CNT 的峰值发射电流由 100\%衰减到 50\%

\section{关键词}

碳纳米管

镍膜

强流脉冲发射

提高稳定性

归一化电流 所经历的发射次数分别约为 3 和 11 次.

碳纳米管 (carbon nanotube, CNT)由于具有纳米 尺度的半径、极高的长径比、出众的机械强度、良好 的导电性能和化学稳定性 ${ }^{[1 \sim 3]}$, 已受到越来越多的关 注. 这些特性使得碳纳米管在许多领域具有应用潜 力, 例如场发射显示器、高分辨电子束仪器、场发射 照明灯、 $X$ 射线源和微波放大管等 ${ }^{[4 \sim 9]}$. CNT 的发射 稳定性, 特别是在强流脉冲发射条件下的发射稳定 性, 是其在电子发射源上应用的关键参数. 但是目前 还没有关于 CNT 强流脉冲发射稳定性的报道.

在本文中, 我们研究了直接生长的 CNT 薄膜在 强流脉冲发射条件下的发射稳定性, 并介绍镍层对 于发射稳定性的改进作用.

\section{1 实验}

CNT 薄膜直接生长在有镍和无镍的 $\mathrm{n}$ 型(100)单 晶硅片上. 所用硅片为单面抛光, 电阻率为 $10^{-2} \sim 10^{-3}$
$\Omega \mathrm{cm}^{-1}$, 直径为 2 英寸. 镍层采用化学镀方法制备. 镀液由硫酸镍、次亚磷酸钠和柠檬酸三钠组成. 镀液 的 $\mathrm{pH}$ 用氨水调至 8 10. 施镀过程中用钯做催化剂. CNT 薄膜采用酞菁铁 $(\mathrm{FePc})$ 高温热解方法制 备 ${ }^{[10]}$. 样品形貌(包括镍层、Si-CNT 薄膜、Ni-CNT 薄膜等) 用扫描电子显微镜(SEM, JEOL JSM-6700F)进行观察 研究. 强流脉冲发射特性测试在脉冲功率系统中采 用二极结构来进行, 系统真空度为 $\sim 5 \times 10^{-4} \mathrm{~Pa}$. 强流 脉冲发射采用单脉冲发射模式, 即每次测试只产生 1 个电压脉冲. 为研究发射稳定性, 我们对 Si-CNT 和 Ni-CNT 阴极在相同主Marx电压下进行重复测量. 每 个电压脉冲的半高宽为 $100 \mathrm{~ns}$.

\section{2 结果与讨论}

\section{1 样品形貌}

图 1 给出了镍膜的 SEM 照片, 其中图 1(b)为(a) 


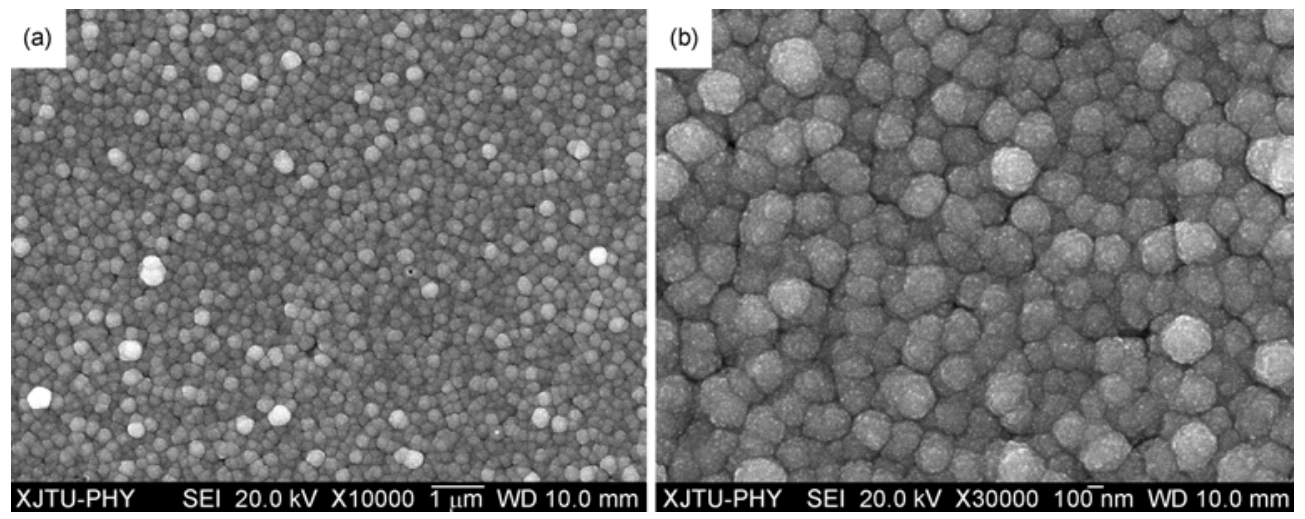

图 1 镍膜的 SEM 照片

的放大照片. 由图 1(a)可以看出, 化学镀方法制备的 镍膜致密而且均匀. 从图 1(b)可以估算出镍颗粒的尺 度约在 150 300 nm 范围内.

Si-CNT 和 Ni-CNT 薄膜的 SEM 照片如图 2 所示, 其中(a)和(b)分别对应于 Si-CNT 和 Ni-CNT. 由图 2 可以看出, 2 种薄膜中 $\mathrm{CNT}$ 生长取向均垂直于基底, 膜层厚度约为几微米.

\section{2 强流脉冲发射电流衰减特性}

尽管发射电流的测量是在相同的主 Marx 电压下 进行的, 但每次输出到阴阳极之间的脉冲电压并不 相同，存在 1 个小幅的波动. 稳定性测试所采用的脉 冲电压峰值的范围为 1.62 1.66 MV, 对应场强为 $11.57 \sim 11.85 \mathrm{~V} / \mu \mathrm{m}$.

图 3 给出了 $\mathrm{Si}-\mathrm{CNT}$ 和 Ni-CNT 阴极的发射电流 重复测量结果. 图 3(b)中第 3 次发射测量数据缺失, 这是由于在该次测试过程中最大电流数据超出记录仪 记录范围所致. 从图3(a)和(b)可见, Si-CNT和 Ni-CNT 阴极首次发射的峰值电流分别为 109.4 和 180.5 A.
随后峰值电流随测量次数(发射序数) 的增加呈现总 体衰减的趋势。在电流测量结果中也发现存在电流 涨落, 这种涨落可能是由于所施加的脉冲电压存在 涨落所致. 这虽然会影响电流测量结果, 但并不会改 变电流衰减的趋势.

由于目前找不到描述强流脉冲发射模式下的电 流衰减公式, 我们参照类似文献[11]中的方法, 用指 数衰减模型来描述 CNT 阴极在强流脉冲条件下的电 流衰减行为. 假设发射电流衰减行为符合一阶指数 衰减规律, 我们可以在 Origin6.1 中用“Fit Exponential Decay”中的 ExpDec 1 模型来进行拟合分析, 并得到 拟合曲线.

ExpDec 1 模型可以描述为

$$
y=y_{0}+A_{1} \mathrm{e}^{-x / t_{1}} .
$$

根据(1)式及在 Origin6.1 中的拟合结果, Si-CNT 和 Ni-CNT 阴极的电流衰减公式可写为

$$
\begin{aligned}
I_{1} & =I_{10}+A_{11} \mathrm{e}^{-x / t_{1}} \\
& =33.75509+169.06883 \mathrm{e}^{-x / 1.27035}(\mathrm{~A}),
\end{aligned}
$$
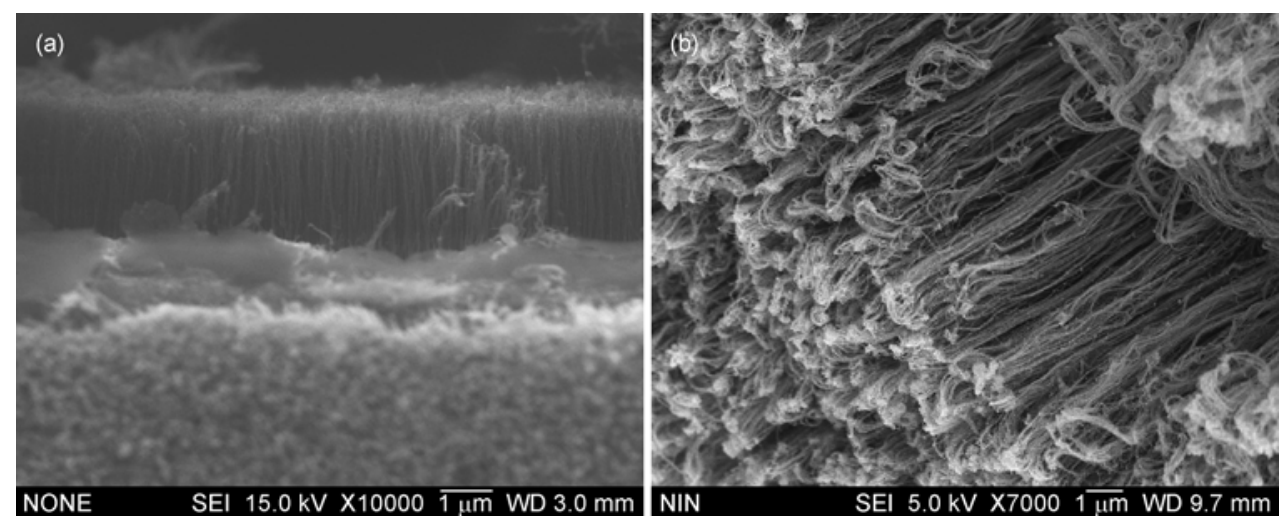

图 2 Si-CNT (a) 和 Ni-CNT (b) 薄膜的 SEM 照片 

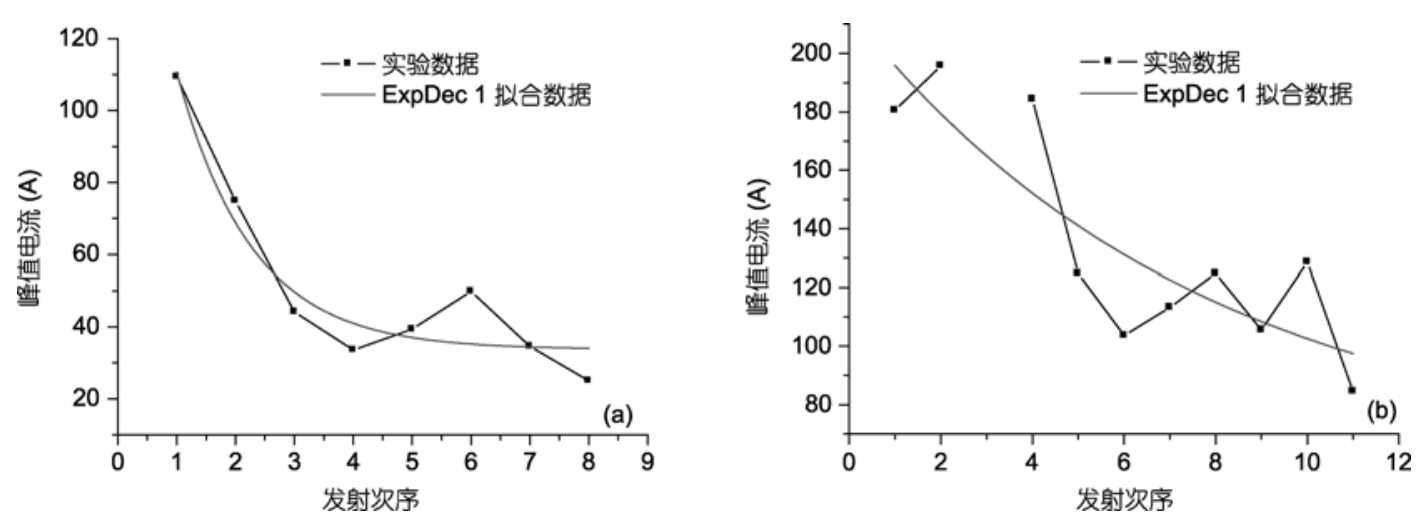

图 3 Si-CNT (a) 和 Ni-CNT (b) 阴极的电流衰减特性

$$
\begin{aligned}
I_{2} & =I_{20}+A_{21} \mathrm{e}^{-x / t_{2}} \\
& =60.69897+153.88518 \mathrm{e}^{-x / 7.69049}(\mathrm{~A}),
\end{aligned}
$$

其中 $I_{1}$ 和 $I_{2}$ 分别为 Si-CNT 和 Ni-CNT 阴极的峰值发 射电流, $x$ 为与 $I_{1}$ 和 $I_{2}$ 相对应的发射次数.

图 3(a)和(b)中的光滑曲线分别表示 Si-CNT 和 $\mathrm{Ni}-\mathrm{CNT}$ 阴极峰值发射电流的一阶指数衰减拟合曲线, 在图中以“ExpDec 1 拟合数据”来表示. 从实验数据 和拟合曲线均不难看出, Ni-CNT 阴极的电流衰减速 度明显低于 Si-CNT 阴极。

\section{3 用归一化电流比较 2 种阴极的电流衰减特性}

考虑到 2 种阴极在发射电流大小上存在差异, 可 以采用归一化峰值电流来描述 2 种阴极的电流衰减 行为, 并比较其衰减速度. 归一化电流定义为每种阴 极在拟合曲线中的各个峰值电流值与该种阴极拟合 曲线中的第 1 个峰值电流值 (亦即拟合曲线中的最大 电流值)的比值. 归一化电流实际上是一个没有量纲 的相对电流, 可由下式给出:

$$
I_{n}=\frac{I}{I_{\mathrm{Max}}},
$$

其中 $I_{n}$ 为归一化电流, $I$ 为拟合曲线中的电流值, $I_{\mathrm{Max}}$ 为拟合曲线的第 1 个电流(最大电流)值.

为了直观地比较 Si-CNT 和 Ni-CNT 阴极发射电流 的衰减趋势, 可将两者的归一化电流曲线放在同一图 中比较. 将归一化电流设为 $Y$ 坐标, 而将发射次序数 值设为 $X$ 坐标, 然后在图中画出两者的归一化电流曲 线. 图 4 给出了 2 种阴极的归一化电流比较曲线, 其中 曲线(a)和(b)分别对应于 Si-CNT 和 Ni-CNT 阴极.

由于归一化电流表示的是归一化曲线中的每个

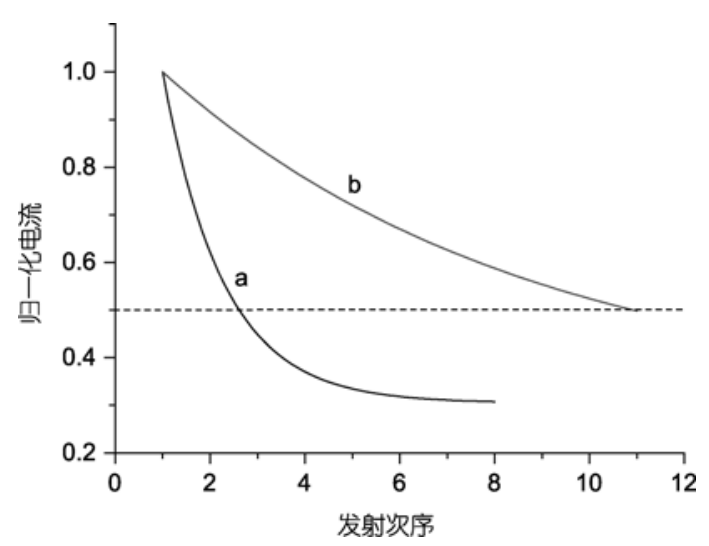

图 4 Si-CNT (a)和 Ni-CNT (b) 阴极发射电流衰减趋势比较

发射电流值相对于第一个发射电流值的百分比, 因 而可以用发射电流衰减到同一个归一化电流所经历 的发射次数来比较电流衰减速度. 如果以 $50 \%$ 作为 标准, 则 Si-CNT 和 Ni-CNT 阴极归一化发射电流由 $100 \%$ 衰减到 50\%所经历的发射次数分别约为 3 和 11 次, 可见镍层的引人对于提高 CNT 阴极的发射稳定 性具有显著的改善作用.

\section{4 发射稳定性改进机理分析}

一般来说，影响常规的直流场发射稳定性的因 素主要包括：CNT 和残留气体分子的表面化学反 应 $^{[12]}$, 以及在发射过程中由于高发射电流产生的巨 大焦耳热对 $\mathrm{CNT}^{[13]}$ 或 CNT-衬底界面 ${ }^{[14]}$ 所造成的损 害. 因此, 用无定形碳钝化 CNT 表面可阻止 CNT 与 周围气体分子的反应 ${ }^{[15]}$, 从而提高发射稳定性. 另 一方面, CNT 同衬底之间良好的结合对于提高发射稳 定性也至关重要 ${ }^{[16]}$, 因为发射过程中作用于 CNT 上 的强大静电力可能将 CNT 从衬底剥离, 从而导致电 流衰减甚至对阴极产生更进一步的伤害. 而且, 良好 
的结合可以减小 CNT-衬底之间的接触电阻, 从而减 少发射过程产生的热量.

在强流脉冲发射模式下, CNT阴极会发射非常高 的发射电流, 因此由于电阻加热效应对阴极造成的 伤害可能是造成电流衰减的主要原因. 镍层的引人 对于提高发射稳定性有两方面的积极作用: (1) 镍层 的电阻小于硅基底的电阻, 这可以减少在电子发射 过程中产生的焦耳热; (2) 根据我们的实验结果, CNT 同镍层之间的结合要好于同硅基底之间的结合, 这可以降低 CNT-界面之间的接触电阻. 这 2 个方面 共同减少了在场发射过程中由于高电流产生的电阻 热效应对于阴极的伤害, 从而使发射稳定性得以提高.

此外, 当 $\mathrm{CNT}$ 生长密度增加时, 由于电场屏蔽 效应的出现会导致开启场强提高和发射电流下降, 从而会导致 CNT 阴极发射能力下降 ${ }^{[17,18]}$. 对于密度 极高的 CNT 薄膜, 只有少数站在薄膜上面的 CNT 才
具备很强的电流发射能力. 这些 CNT 在发射过程中 受到损害的可能性就会大大提高, 特别是在强流脉 冲发射模式下. 因此, 如果 CNT 的生长密度能控制 在适当的范围内, 可能会使 CNT 阴极的电流发射能 力和发射稳定性有进一步的提高.

\section{3 结论}

采用酞菁铁高温热解方法制备了 $\mathrm{Si}-\mathrm{CNT}$ 和 Ni-CNT 两种阴极, 其中镍层采用化学镀方法制备. 对于 2 种阴极在强流脉冲发射模式下的发射稳定性 测试结果表明, 镍层的引人可以显著提高 CNT 阴极 的发射稳定性. 对 2 种阴极重复发射的归一化电流进 行比较, 可以得到更直观的改进效果：Si-CNT 和 Ni-CNT两种阴极的归一化峰值电流从 $100 \%$ 衰减到 $50 \%$ 所经历的发射次数分别约为 3 和 11 次, Ni-CNT 阴极发射电流的衰减速度明显低于 Si-CNT 阴极.

\section{参考文献}

1 Iijima S. Helical microtubes of graphitic carbon. Nature, 1991, 354: 56-58

2 De Heer W A, Chatelain A, Ugarte D. A carbon nanotube field emission electron source. Science, 1995, 270: 1179-1180

3 Bonard J M, Croci M, Klinke C, et al. Carbon nanotube films as electron field emitters. Carbon, 2002, 40: 1715-1728

4 de Jonge N, Bonard J M. Carbon nanotube electron sources and applications. Philos Trans R Soc London A, 2004, 362: 2239-2266

5 de Jonge N, Lamy Y, Schoots K, et al. High brightness electron beam from a multi-walled carbon nanotube. Nature, 2002, 420: 393-395

6 Teo K B K, Chhowalla M, Amaratunga G A J, et al. Fabrication and electrical characteristics of carbon nanotube-based microcathodes for use in a parallel electron-beam lithography system. J Vac Sci Technol B, 2003, 21: 693-697

7 Cheng Y, Zhang J, Lee Y Z, et al. Dynamic radiography using a carbon-nanotube-based field-emission X-ray source. Rev Sci Instrum, 2004, 75: 3264-3267

8 Choi W B, Chung D S, Kang J H, et al. Fully sealed, high-brightness carbon-nanotube field-emission display. Appl Phys Lett, 1999, 75: 3129-3131

9 Teo K B K, Minoux E, Hudanski L, et al. Microwave devices: Carbon nanotubes as cold cathodes. Nature, 2005, 437: 968

10 Li D C, Dai L M, Huang S M, et al. Structure and growth of aligned carbon nanotube films by pyrolysis. Chem Phys Lett, 2000, 316 : 349-355

11 Weon B M, Je J H. Time-dependent current-voltage relation in electron guns. J Appl Phys, 2005, 97: 036101

12 Dong C, Gupta M C. Influences of the surface reactions on the field emission from multiwall carbon nano-tubes. Appl Phys Lett, 2003, 83: $159-161$

13 Nilsson L, Groening O, Groening P, et al. Collective emission degradation behavior of carbon nanotube thin-film electron emitters. Appl Phys Lett, 2001, 79: 1036-1038

14 Rao A M, Jacques D, Haddon R C, et al. In situ-grown carbon nanotube array with excellent field emission characteristics. Appl Phys Lett, 2000, 76: 3813-3815

15 Jo S H, Tu Y, Huang Z P, et al. Correlation of field emission and surface microstructure of vertically aligned carbon nanotubes. Appl Phys Lett, 2004, 84: 413-415

16 Cheng Y, Zhou O. Electron field emission from carbon nanotubes. CR Phys, 2003, 4: 1021-1033

17 Choi J H, Choi S H, Han J H, et al. Enhanced electron emission from carbon nanotubes through density control using in situ plasma treatment of catalyst metal. J Appl Phys, 2003, 94: 487-490

18 Kong B Y, Seon J Y, Lee S H, et al. Density control of highly populated carbon nanotubes grown by thermal chemical vapor deposition to improve their field emission characteristics. J Korean Phys Soc, 2004, 45: 1580-1583 